\title{
New initiative: "Ten Questions in Sports Engineering" papers
}

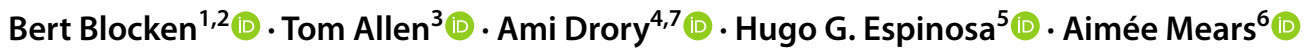

Accepted: 10 August 2021 / Published online: 23 August 2021

(c) International Sports Engineering Association 2021

The journal Sports Engineering launches a new publication initiative: "Ten Questions in Sports Engineering” papers. Ten questions papers should deal with a well-defined topic and should be centered around a selection of ten relevant and topical questions. Submissions are based on invitation only by an (Associate) Editor of the journal.

\section{Brief description of the initiative}

Sports Engineering is launching a new initiative for a series of "Ten Questions in Sports Engineering" papers, to be published in a pre-described format. The concept of the "Ten Questions" papers was launched in 2015 for the journal Building and Environment [1]. Some examples of published Ten Questions papers can be found here [2-5]. The concept is now also implemented for Sports Engineering, with approval from the Editors-in-Chief of Building and Environment. "Ten Questions in Sports Engineering" papers should deal with a well-defined topic and should be centered around a selection of ten relevant and topical questions. Submissions are based on invitation only by an (Associate) Editor

Bert Blocken

b.j.e.blocken@tue.nl

1 Department of the Built Environment, Eindhoven University of Technology, Eindhoven, The Netherlands

2 Department of Civil Engineering, KU Leuven, Leuven, Belgium

3 Department of Engineering, Manchester Metropolitan University, Manchester, UK

4 College of Engineering and Computer Science, Australian National University, Canberra, Australia

5 School of Engineering and Built Environment, Griffith University, Brisbane, Australia

6 School of Mechanical, Electrical and Manufacturing Engineering, Loughborough University, Loughborough, UK

7 National Facility for Human Robot Interaction Research, University of New South Wales, Sydney, Australia of the journal, and if published they will be promoted as invited papers. Papers will be recruited in a way that ensures balanced coverage of all topics in the scope of Sports Engineering. The papers should be (co-)authored by established researchers in the field with proven expertise on the topic, typically by an extensive track record of published books and peer-reviewed journal papers. The questions are selected and answered by the author(s). The questions should be such that the paper is visionary, authoritative and can provide early career researchers directions for future research.

The submission and review procedure is outlined in detail in Sect. 2.

\section{Submission and review procedure}

"Ten Questions in Sports Engineering" papers will undergo a two-step review process.

\subsection{Step 1}

Invited authors will submit the following components of the intended paper to the (Associate) Editor that invited them to submit a "Ten Questions in Sports Engineering" paper:

- title

- abstract (max. 250 words)

- keywords

- tentative list of ten questions

- one-sentence answers to the questions

- description of expertise of the authors on the topic (max. 250 words)

- list of at least five suggested reviewers (name, title, affiliation, email address, reason for selection)

- intended submission date of full paper

The title of every paper should be similar and should start with the words: "Ten questions in Sports Engineering: ...". Two examples: 
- "Ten questions in Sports Engineering: Wearable sensors"

- "Ten questions in Sports Engineering: Drafting in cycling"

The questions should be as concise and as clear as possible. The (Associate) Editor will evaluate whether the proposed contribution fits the scope and requirements of the series of "Ten Questions in Sports Engineering" papers and whether it overlaps with a published review or other papers in Sports Engineering or other journals. If evaluated positively, the (Associate) Editor will already select at least two expert reviewers, some of which might be selected from the list of at least five suggested reviewers. The entire process will be conducted in double-blind peer review. The reviewers are welcome to improve the formulation of the questions or to suggest some other questions for consideration by the author(s). The same reviewers will afterwards evaluate the full paper. This first and preliminary review stage is handled outside the journal's online submission system, to reduce the effort needed by the author(s) in revising the full paper afterwards.

\subsection{Step 2}

The author(s) will submit the revised set of components using the journal's online submission system, together with a detailed response to reviewers, addressing every point raised by them. The full paper must not exceed 4000 words. The mandatory structure of every "Ten Questions in Sports Engineering" paper is given in the Appendix.

The maximum number of figures is 10 . The maximum number of tables is 5 . The introduction should address the reasons for selecting these specific questions. The answers to the questions should satisfy the requirements of original review or other papers. Authors should take care to include not only their own work but also relevant work by others in the answers to the questions. New research results cannot be introduced in the paper, as this is not the purpose of this type of paper and its limited length does not allow full description of the research that has led to the new results. Where the author(s) provide their personal view on the topic without support by literature references, this should be clearly indicated as such. Apart from the above-mentioned requirements, the "Ten Questions in Sports Engineering" papers must also adhere to the regular requirements stated in the Sports Engineering Submission Guidelines on the journal website and in the editorial "How to write a manuscript for Sports Engineering" [6]. The author(s) submit the full paper by means of the online submission system by selecting the option "Invited paper" in the submission drop-down list. The paper will be forwarded to the anonymous expert reviewers that were already selected in the preliminary review stage in step 1 . This second part of the review process is similar to that for regular original research papers.

\section{Appendix}

Mandatory structure of a "Ten Questions in Sports Engineering" paper:

Abstract

1. Introduction

2. Question 1: ...

Answer to question 1.

3. Question 2: ...

Answer to question 2.

4. Question 3: ...

Answer to question 3.

5. Question 4: ...

Answer to question 4.

6. Question 5: ...

Answer to question 5.

7. Question 6: ...

Answer to question 6 .

8. Question 7: ...

Answer to question 7.

9. Question 8: ...

Answer to question 8 .

10. Question 9: ...

Answer to question 9.

11. Question 10: ...

Answer to question 10.

12. Discussion

13. Conclusion

References

\section{References}

1. Blocken B (2015) New initiative: ten questions paper series in building and environment. Build Environ 94(1):325-326

2. Meroney RN (2016) Ten questions concerning hybrid computational/physical model simulation of wind flow in the built environment. Build Environ 96:12-21

3. Steinemann A, Wargocki P, Rismanchi B (2017) Ten questions concerning green buildings and indoor air quality. Build Environ 112:351-358

4. Derome D, Kubilay A, Defraeye T, Blocken B, Carmeliet J (2017) Ten questions concerning modeling of wind-driven rain in the built environment. Build Environ 114:495-506

5. Hong T, Chen Y, Luo X, Luo N, Lee SH (2020) Ten questions on urban building energy modelling. Build Environ 168:106508

6. Allen T, Blocken B, Drory A, Espinosa HG (2020) How to write a manuscript for Sports Engineering? Sports Eng 23:23

Publisher's Note Springer Nature remains neutral with regard to jurisdictional claims in published maps and institutional affiliations. 